\title{
Moneda y territorio en Al-Andalus: Un ejemplo de hallazgos en Jaén
}

Alberto Canto García*

\section{INTRODUCCIÓN}

Es algo conocido en el terreno de la numismática la profunda relación que existe entre la producción de monedas y el territorio por el que se desenvuelven y la información que esta simbiosis nos proporciona sobre aspectos muy importantes de la vida económica y política de una sociedad. La existencia de diferentes talleres o cecas y sus áreas predominantes de circulación muestran tendencias y pueden manifestar corrientes y flujos de intercambios entre ellas, al tiempo que la presencia de monedas procedentes de centros de emisión más alejados nos pueden indicar relaciones más complejas todavía (BARCELO, 1988).

\section{El modelo omeya en al-Andalus}

La producción de moneda en al-Andalus, sobre todo para el periodo omeya y el de las primeras taifas del siglo $\mathrm{Xl}$, aparece como uno de los hilos conductores de la investigación en aspectos en los que, por diversas circunstancias, nos falta una información más fiable al respecto; de hecho podríamos decir que la moneda es el único elemento de interpretación (en el estado actual de nuestros conocimientos) para analizar ciertos fenómenos.

Es cierto que el planteamiento anterior parece lógico y corroborado por una fuerte tradición de investigación numismática e histórica, pero el caso concreto de al-Andalus sus- cita problemas muy peculiares, sobre todo para los primeros siglos de la historia islámica en la Península Ibérica.

La estructura de la producción monetaria andalusí se articuló, desde época muy temprana en torno a un único centro de producción, que desde época de cAbd al-Raḥmān I, sabemos corresponde a Córdoba, aunque aparezca englobado bajo el nombre genérico de alAndalus. Así, desde una época temprana, todas las emisiones del estado omeya llevarán el mismo nombre de ceca, sin ninguna concesión a la presencia de otros nombres, con excepción hecha de la aparición (en época del califa cAbd al-Raḥmān III) del nombre de la ciudad palatina de madīnat al-Zaḥrā', en sustitución de al-Andalus, hecho este que obedece al traslado del taller de moneda desde la ciudad de Córdoba a la nueva residencia califal.

Esta concentración de la producción en un único taller, de forma casi total, elimina toda posibilidad de análisis de circulación entre talleres diferentes (salvo la excepción de la presencia de monedas procedentes de los talleres africanos del territorio bajo control omeya, como Fās, Nakūr o Siŷilmasā ).

Solo resta el estudio de los hallazgos monetarios y su distribución dentro del territorio de al-Andalus como fuente para interpretar la evolución y el grado e intensidad de la presencia de moneda en las ciudades y terrenos andalusíes.'

\footnotetext{
* Dpto. de Prehistoria y Arqueología, Facultad de Filosofía y Letras, U. Autónoma de Madrid

I En cualquiera de los casos la interpretación de hallazgos está sujeta al número de los mismos que conocemos y cuya información podemos contrastar.
} 
Ahora bien, una breve revisión de la evolución de los hallazgos obliga a entender cuál es el mecanismo de funcionamiento del sistema monetario que los omeyas trajeron a alAndalus. Pasado el momento de la invasión en el que las conversiones en numerario del botín obtenido fueron altas y la dinámica de la conquista continuaba su proceso, caracterizado por emisiones de dinares y dirhemes, se produce un descenso de la actividad monetaria en los años correspondientes a la crisis política que sacude al-Andalus a mediados del siglo VIII, y que tendría su solución con la venida de cAbd al-Rahmān I.

Desde este momento el emirato omeya de Córdoba organiza su política fiscal dentro de la cual la moneda será una herramienta indispensable para su funcionamiento (BARCELO, 1985).

Aquí es donde es posible percibir ciertas diferencias entre los hallazgos de los distintos momentos dentro de la época omeya. Si para el periodo de conquista es posible percibir cómo los hallazgos se distribuyen de una manera más uniforme por todo el espacio de al-Andalus, el periodo emiral empieza a mostrar ciertas tendencias que se verán magnificadas en la época del califato.

Esta tendencia se plasma en la abundancia de hallazgos (tanto en cantidad como en tamaño) en ciertas zonas de al-Andalus, que podríamos considerar como interiores o nucleares respecto de la capitalidad omeya, mientras que otras zonas de la periferia del estado omeya muestran una notable ausencia de hallazgos de cierta entidad.

Esta diferencia entre los distintos territorios de al-Andalus puede obedecer a diferentes motivos, pero la tradición del centralismo estatal que los omeyas habían practicado en Oriente también tiene su reflejo en la organización estatal que ponen en pie en al-Andalus, reflejado tanto en el único taller como en la concentración de monedas en los entornos del núcleo del poder omeya cordobés.

Esta tendencia se acentúa en la época del califato, periodo en el que conocemos alguno de los mayores hallazgos de moneda de plata de toda la moneda andalusí (SAENZ-DIEZ, 1984), localizados en el entorno de la ciudad de Córdoba.

Concluyendo podríamos decir que el sistema monetario omeya, parte sustancial y elemento de primer orden dentro del sistema fiscal elaborado por los omeyas, se distribuye por el territorio de al-Andalus de una manera desigual (por definirlo de alguna forma), con profundas diferencias entre la cantidad de monedas encontradas en el alfoz de Córdoba y algunas otras ciudades importante de alAndalus, respecto de otras zonas del territorio andalusí en las que el elemento monetal se caracteriza por su ausencia.

La transición del mundo omeya a la época taifa del siglo $\mathrm{XI}$ supone un cambio radical para el panorama de los hallazgos (CANTO 1992). De una forma totalmente distinta la dispersión de los hallazgos y su distribución por los nuevos reinos y poderes políticos que surgen en al-Andalus constituye un modelo de uso de moneda muy diferente al del periodo anterior (BARCELÓ 1992 y CANTO 1994).

En el Museo de Jaén se encuentran depositados dos hallazgos que ilustran de manera muy representativa este periodo de los comienzos de las emisiones taifas. Ambos hallazgos aparecieron en una misma zona y de ahí la denominación de caracter geográfico utilizada para identificarlos entre ellos. La similitud de sus composiciones internas nos hacen sospechar que pueda tratarse de un mismo hallazgo, aunque no tengamos constancia de ello. Por ellos he decidido mantener los dos lotes separados y analizarlos de forma individual, aunque los datos proporcionados pueden confrontarse con facilidad.

Estos dos conjuntos son una clara muestra del proceso de descentralización de moneda que se produce en el siglo XI d.C. La presencia de piezas de dos dinastías, con certeza, y de un grupo anónimo (posiblemente asignable a una de las dinastías presentes en los conjuntos), convierte al conjunto en algo bastante homogéneo, con poca dispersión . 


\section{Hallazgo de Marroquíes Altos I (Jáen).}

\section{Descripción}

Consiste en un lote de monedas del periodo taifa, localizado en uno de los actuales barrios de la ciudad de Jaén, cerca del lugar donde está situado en la actualidad el Museo Arqueológico Provincial. Cabe la posibilidad de que formase parte de un segundo hallazgo de este Museo, que hemos denominado "Marroquíes Altos 2", y que se encontró en la misma zona; dado que en los fondos del Museo se encontraban separados, y con números de Inventario que no eran secuenciales, hemos optado por mantenerlos separados, aunquehabría que considerar seriamente la opinión que considera a ambos, como procedentes de un mismo conjunto.

\section{Análisis}

El hallazgo se compone de fracciones de dinar de los ziríes de Granada, (núms. I-2), de los Banū Yahwar de Córdoba (núms.3-I8), un grupo anónimo, que consideramos, asimismo, de esta última dinastía (núms. 19-33) y una última moneda anónima (la número 34).

Como se indica en el catálogo, la similitud entre la serie de los Banū Yahwar, y las anónimas nos hace creer que puedan pertenecer a la misma dinastía, pues ambos grupos emiten a nombre de cAbd Allah. La última moneda, con fecha más tardía, tambíen podría ser de la misma dinastía, pero es más difícil de atestiguar.

El gráfico núm. I nos muestra la proporción existente dentro del hallazgo, siendo la dinastía de los Banū Yahwar la más numerosa con un $91,18 \%$ del total, seguido por los ziríes con el 5,88 \% y la última moneda anónima representa el 2,94\%.

En el grupo mayoritario hemos englobado juntas a las anónimas, que representan la mitad menos uno de los ejemplares de los Banū Yahwar; si lo separamos vemos que el grupo de los Banū Yahwar supone el 47,06\%, seguido de las anónimas con un 44,12\%, los zirríes con el 5,88\% y la última anónima con el 2,94\%. De todas formas creemos que, por similitud en las leyendas y coherencia del conjunto, deben incluirse en un solo grupo.

Es evidente que representan una novedad, esta serie anónima, en tanto que no está recogida en ninguna de las clásicas obras o repertorios, aportando una nueva serie a las emisiones del periodo taifa andalusí. De hecho, como hemos indicado arriba, la mayor similitud la encontramos en alguna de las emisiones de los Banū Yahwar, que situan en sus monedas la leyenda

\section{الامام عبد الله}

en dos líneas en el anverso de la moneda, disposición que resulta muy similar a la adoptada por estas monedas aunque repartida en ambas caras de la moneda. Algo similar ocurre con la última moneda del conjunto, que podría ser también de los Banū Yahwar; en este caso el modelo es muy similar a emisiones de Córdoba, aunque Prieto y Vives ubicase a ejemplares con esta distribución de leyendas dentro de las monedas anónimas de alAndalus, ${ }^{2}$ aunque las fechas de los ejemplares descritos por Prieto, son de los primeros años del siglo $\mathrm{V} \mathrm{H}$., y la moneda que nos ocupa tiene una datación mucho más tardía, que la podría vincular a la serie de los Banū Yahwar.

\section{Metrología ${ }^{3}$}

En cuanto a la metrología de este hallazgo sólo nos hemos ocupado del grupo de los Banū Yahwar, por ser el único que aporta un grupo sustancial de monedas para este tipo de análisis. Uno de los problemas de la metrología de las series taifas radica en la variedad, o mejor dicho en el desconocimiento del patrón o valor al que responden determinadas emisiones, que por tradición (o comodidad) se denominan como tercios o fracciones de dinar. Sólo el estudio sistemático

2 A. Prieto (1926), pp. 146-7 y 240, núm. 437a, b yc.

3 Conviene recordar que el número de ejemplares utilizado es escaso, pero hemos mantenido el análisis por coherencia del hallazgo; es evidente que a mayor número de monedas de los determinados períodos, más seguras serán las conclusiones estadísticas. 
de sus pesos y de las frecuencias de los mismo podría ayudar a determinar un valor real a todas estas series.

En el grupo de monedas que hemos considerado como de los Banū Yahwar, se integran 3 I ejemplares, con los siguientes resultados:

$\times$ 0,74

Desviación Típica. 0,17

Error típico $.0,03$

Varianza. 0,03

Coef. de variación $.24 \%$

Kurtossis $-0,39$

Skewness $.0,04$
Este análisis nos muestra algunas de las características del periodo, como un coeficiente de variación bastante alto, el $24 \%$, que nos indica una irregularidad en los pesos de las emisiones, y los parámetros de simetría (skewness) y aplanamiento (kurtosis) indican que los valores no son simétricos, en el caso del primero, y en lo que respecta al segundo, que predominan las frecuencias más bajas que las que corresponde a su normalidad teórica.

En suma, existen ciertas alteraciones que son un síntoma de las irregularidades que afectan a las emisiones de los Banū Yahwar, que por otra parte son comunes a otras emisiones del periodo de las taifas.

\section{CATÁLOGO}

Zīríes de Granada

Bādīs b. Habūs (430-467H.) M. Garnāța

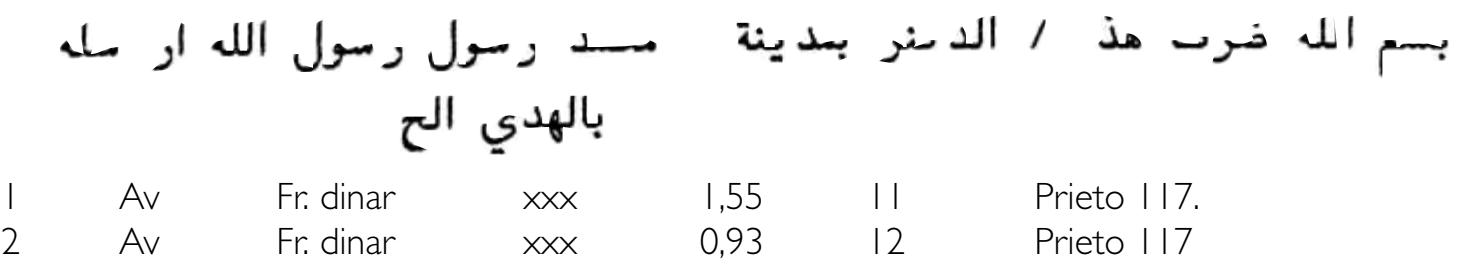

Banū Yahwar de Córdoba

Abūl-Walìd Muḥammad b. Yahwar (434-456 H.) (Qurțuba )
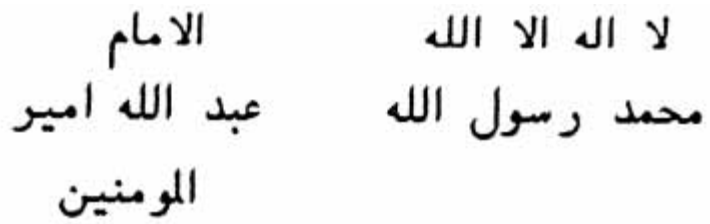

Corán 61.9 Ley. de ceca y fecha

3 Av Fr. dinar $\quad x x x \quad 0,62 \quad$ I $\quad$ Prieto 352 var.; Vives |39|. Las monedas citadas por Prieto, corresponden a los años $440-44 \mathrm{IH}$., las que aquí recogemos no presentan fechas claras de lectura.

\begin{tabular}{|c|c|c|c|c|c|c|}
\hline 4 & Av & Fr. dinar & $x x x$ & 0,68 & 10 & Prieto 352 var:; Vives I39| \\
\hline 5 & $A v$ & Fr. dinar & $x x x$ & 0,83 & 10 & Prieto 352 var:; Vives I39| \\
\hline 6 & Av & Fr. dinar & $x x x$ & 0,86 & | | & Prieto 352 var:; Vives I3s \\
\hline 7 & Av & Fr. dinar & $x x x$ & 1,14 & 12 & Prieto 352 var:; Vives 13 \\
\hline 8 & Av & Fr. dinar & $x x x$ & 0,92 & 12 & Prieto 352 var. Vives I 39 \\
\hline 9 & Av & Fr. dinar & $x x x$ & 1,05 & 12 & Prieto 352 var:; Vives 13 \\
\hline 10 & Av & Fr. dinar & $x x x$ & 0,58 & | | & Prieto 352 var:; Vives 13 \\
\hline | | & Av & Fr. dinar & $x x x$ & 0,82 & | & Prieto 352 var:; Vives I 39 \\
\hline
\end{tabular}




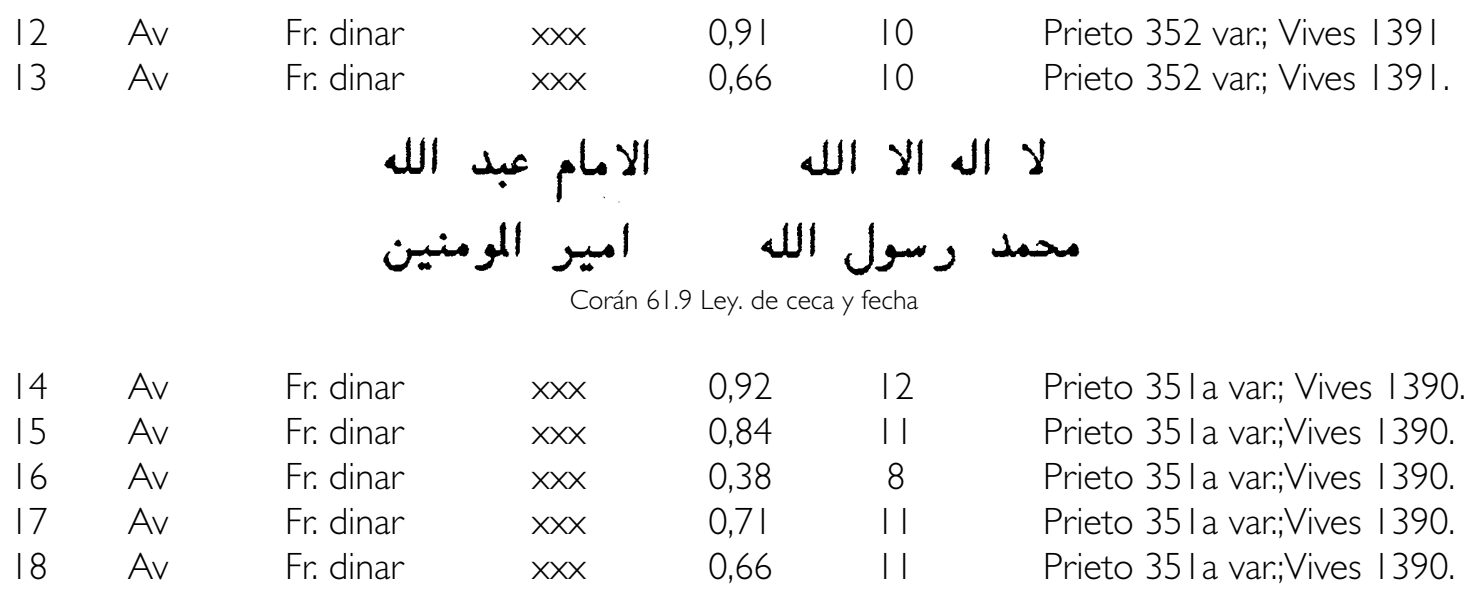

\section{Anónimas}

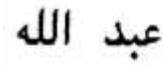

19
Av

Fr. dinar

$X X X$

0,89

المام

La serie inédita presenta cuatro variantes en los modelos de decoración. No está recogida en la bibliografía existente y podria pertenecer a los Banū Yahwar, por coherencia con el resto del conjunto y por las similitu des que la distribución de las leyendas tiene con el resto de la serie de los citados Banū Yahwar.

\begin{tabular}{|c|c|c|c|c|c|c|}
\hline 20 & Av & Fr. dinar & $x x x$ & 0,50 & 10 & Inédita \\
\hline 21 & Av & Fr. dinar & $x x x$ & 0,80 & II & Inédita \\
\hline 22 & $A v$ & Fr. dinar & $x x x$ & 0,69 & | | & Inédi \\
\hline 23 & $A v$ & Fr. dinar & $x x x$ & 0,62 & 9 & Inédita \\
\hline 24 & Av & Fr. dinar & $x x x$ & 0,80 & 9 & Inédi \\
\hline 25 & Av & Fr. dinar & $x x x$ & 0,51 & 9 & Inédi \\
\hline 26 & Av & Fr. dinar & $x x x$ & 0,78 & 10 & Inéd \\
\hline 27 & Av & Fr. dinar & $x x x$ & 0,85 & 9 & Inéd \\
\hline 28 & Av & Fr. dinar & $x \times x$ & 0,44 & 9 & Inéd \\
\hline 29 & Av & Fr. dinar & $x \times x$ & 0,56 & 10 & néd \\
\hline 30 & Av & Fr. dinar & $x \times x$ & 0,71 & 10 & Inédi \\
\hline 31 & $A v$ & Fr. dinar & $x x x$ & 0,58 & 8 & Inéd \\
\hline 32 & Av & Fr. dinar & $x x x$ & 0,73 & 9 & Inéd \\
\hline 33 & Av & Fr. dinar & $x x x$ & 0,97 & 10 & Inéd \\
\hline
\end{tabular}

\section{Anónimas (al-Andalus )}
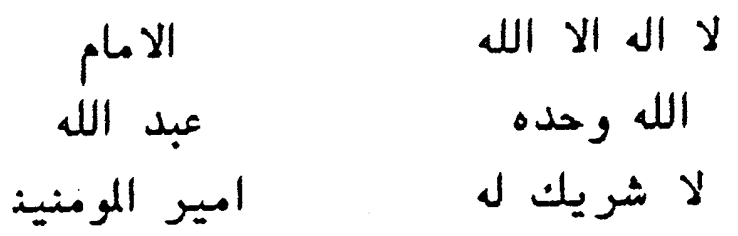

Corán 61.9 Ley. de ceca y fecha

34 Av $\quad$ Fr. dinar $\quad 443 \quad 0,95 \quad 12 \quad$ Prieto 437; Vives 1397 


\section{Hallazgo de Marroquies Altos 2 (Jaén)}

\section{Descripción}

Como hemos indicado en el hallazgo anterior, este segundo lote de monedas aparece como localizado en la misma zona, y su composición es muy similar al anterior, tanto en las dinastías representadas, como en el tipo y clase de las monedas que lo forman; debido a ello y como indicamos en el anterior hallazgo, existe la posibilidad (muy grande a nuestro juicio) de que formen un único conjunto, pero a falta de pruebas más concluyentes hemos optado por respetar la división existente en la documentación del Museo.

\section{Análisis}

Está compuesto por ejemplares de los ziríes de Granada, de los Banū Yahwar de Córdoba, y una serie de monedas clasificadas como anónimas, pero que por las razones expuestas en el hallazgo anterior, suponemos formen parte de las emisiones de la última dinastía citada.

Son en total 274 monedas, de las cuales I 79 (el 65,33\%) pertenecen a los ziríes; 95 (el 34,67\%) a los Banū Yahwar de Córdoba., como pueden verse en la Figura 2.

En el caso del primer grupo todas ellas responden al modelo de monedas con leyendas concéntricas en ambas caras, pertenecientes a Bādīs, como gobernante independiente de los hammūdíes; aparecen en dos versones diferentes de leyendas, conocidas y recogidas en la bibliografía existente. ${ }^{4}$

En lo que atañe al segundo grupo, está compuesto por dos series de monedas, la primera de ellas, del número 180 al 207, responde a un tipo conocido de ejemplares de AbūI-Walīd Muhammad, ya recogidos por Prieto. ${ }^{5}$ Desde el ejemplar 208 hasta el 274, corresponden a un modelo de moneda inédita que en su anverso es similar a la descrita por Prieto con el número 352, y en su reverso lo es a la Prieto 353. El hecho de reunir los dos diseños en un nuevo tipo, me inclina a asignar esta serie a los Banū Yahwar, sin dudas.

\section{Metrología}

El análisis metrológico de este conjunto es más coherente que el del anterior hallazgo, pues el número de ejemplares es significativamente más amplio.

La serie zirí, con una población de 179 ejemplares proporciona los siguientes resultados:

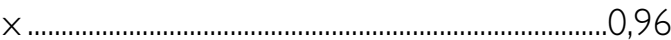

Desviación Típica................................................0,29

Error típico .......................................................... 0,02

Varianza................................................................ 0,08

Coef. de variación .............................................29,8\%

Kurtossis .............................................................

Skewness............................................................... 0,93

En este conjunto los resultados son bastante más coherentes, pues aunque mantiene un coeficiente de variación muy alto, el parametro de simetría se acerca bastante a los valores normales de "0" para la Skewness, y en el valor de la kurtosis (que sería "3" en una muestra normal) indica una clara tendencia hacia valores más bajos de lo normal (Figura 3)

Se aprecia que en torno a la media teórica de peso, los valores se agrupan más hacia la izquierda, con predominio de los valores menores o de peso más reducido, no aparentando una simetría correcta.

Estas variaciones se aprecian, también en la Figura 4, que recoge la frecuencia de pesos de las monedas

Aunque se establece una simetría y una agrupación respecto de los valores centrales, aparece una clara presencia de elementos superiores, que en alguna ocasión, incluso

\footnotetext{
4 Los números 116 y 117 de A. Prieto (1926), pp 176.

5 Número 352 del mismo autor, pp. 220.
} 
duplican los pesos medios teóricos. De todas formas, y ante una muestra numerosa,como es la que aquí tratamos, sobre todo en el periodo de taifas, parece claro que los valores se asemejan a teóricos cuartos de dinar en lo que atañe a su aspecto metrológico (al margen de los estudios de fineza del metal).

Para la serie de los gobernantes de Córdoba, el número de ejemplares es muy superior al utilizado en el anterior hallazgo, y por lo tanto los resultados son algo más fiables; sobre una muestra de 95 monedas los resultados son los siguientes: $x$.

Desviación Típica. $.0,22$

Error típico 0,02

Varianza 0,53

Coef. de variación. $29,7 \%$

Kurtossis. $-0.06$

Skewness 0,64

Para esta serie de los Banū Yahwar, y a pesar de que cuantitativamente sea más fiable, los resultados gráficos que nos muestra el histograma son bastante irregulares, pues nos encontramos ante un conjunto con grandes variaciones en torno a los valores mediales.

Algo similar se aprecia en el análisis de la frecuencia de pesos de la figura 12, que si bien muestra cierta tendencia normal de los valores inferiores a la media, no ocurre lo mismo con la parte superior de la secuencia que muestra profundas alteraciones.

Dichas alteraciones se marcan más en los valores más altos con un desarrollo bastante irregular. En general el peso medio y todo el aspecto del conjunto resulta inferior al mostrado por el grupo de la moneda zirrí.

En este caso podemos ver que, si comparamos la tabla de análisis estadístico con la del hallazgo de "Marroquíes I" correspondiente al mismo tipo de monedas, el peso medio $(x)$ de los ejemplares muestra escasa diferencia entre un 0,77 y el 0,74; los restante valores no son muy diferentes y son más bien reflejo de las características propias de las emisiones del periodo.

Como consideración general resulta dificil aplicar una denominación concreta de valor a estas emisiones, pues su peso es menor de un tercio o un cuarto de dinar (respecto de los baremos califales omeyas). En el caso de las emisiones ziríes debemos tener en cuenta también, que algunos ejemplares no parecen ser de oro de muy buena ley, sino de una aleación que incluye cobre o plata, acercándose más al electro. ${ }^{6}$

\section{CATÁLOGO}

Ziríes de Granada

Badis b. Habus (430-467H.) M. Garnāța

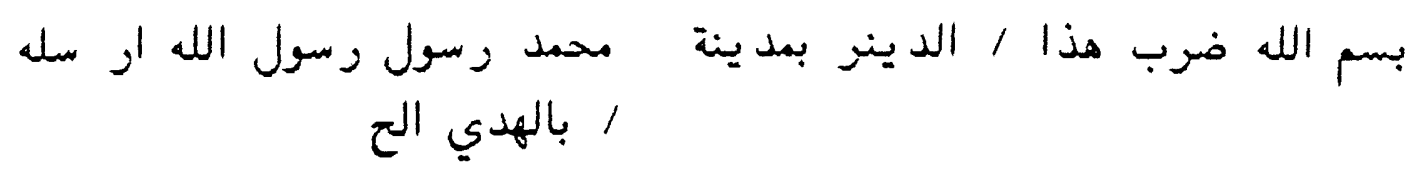

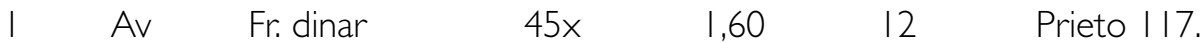

Se ve parte de la leyenda de fecha, estando borrosa la unidad, que podría ser un uno.

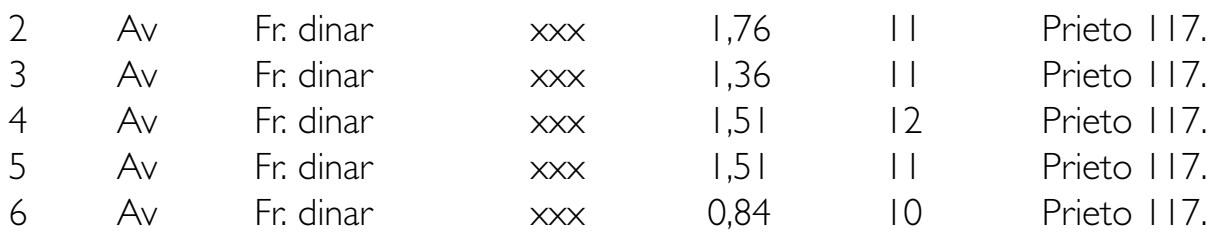

${ }^{6}$ Este problema sólo podrá solucionarse mediante los análisis preceptivos del metal de las monedas, para determinar su composición y valor real. 


\begin{tabular}{|c|c|c|c|c|c|c|}
\hline 7 & $A v$ & Fr. dinar & $x \times x$ & 0,88 & 8 & Prieto 117 \\
\hline 8 & $A v$ & Fr. dinar & $45 x$ & 1,37 & 11 & Prieto 117 \\
\hline 9 & $A v$ & Fr. dinar & $45 x$ & 0,93 & 10 & Prieto 117 \\
\hline 10 & $A v$ & Fr. dinar & $x x x$ & 1,26 & 10 & Prieto 117 \\
\hline | | & $A v$ & Fr. dinar & $x x x$ & 0,42 & 7 & Prieto 117 \\
\hline 12 & AV & Fr. dinar & $x \times x$ & 1,02 & 10 & Prieto 117 \\
\hline 13 & Av & Fr. dinar & $x \times x$ & 0,84 & 9 & Prieto 117 \\
\hline 14 & $A v$ & fr. dinar & $x x x$ & 0,98 & 10 & Prieto 117 \\
\hline 15 & $A v$ & fr. dinar & $x x x$ & 1,26 & 12 & Prieto 117 \\
\hline 16 & $A v$ & Fr. dinar & $x x x$ & 1,17 & 10 & Prieto 117 \\
\hline 17 & Av & Fr. dinar & $x x x$ & 0,64 & 9 & Prieto 117 \\
\hline 18 & $A v$ & Fr. dinar & $x x x$ & 0,95 & 9 & Prieto 117 \\
\hline 19 & Av & Fr. dinar & $x \times x$ & 1,06 & 10 & Prieto 117 \\
\hline 20 & $A v$ & Fr. dinar & $x \times x$ & 0,90 & 9 & Prieto 117 \\
\hline 21 & $A v$ & Fr. dinar & $x \times x$ & 1,27 & 11 & Prieto 117 \\
\hline 22 & $A v$ & Fr. dinar & $x x x$ & 1,15 & 10 & Prieto 117 \\
\hline 23 & Av & Fr. dinar & $x x x$ & 0,56 & 8 & Prieto 117 \\
\hline 24 & Av & Fr. dinar & $x x x$ & 1,24 & 11 & Prieto 117 \\
\hline 25 & Av & Fr. dinar & $x \times x$ & 1,30 & 11 & Prieto 117 \\
\hline 27 & $A v$ & Fr. dinar & $x \times x$ & $0,7 \mid$ & 10 & Prieto 117 \\
\hline 27 & $A v$ & Fr. dinar & $x \times x$ & 0,92 & 10 & Prieto 117 \\
\hline 28 & $A v$ & Fr. dinar & $x \times x$ & 1,52 & 10 & Prieto 117 \\
\hline 29 & Av & Fr. dinar & $x x x$ & 1,08 & II & Prieto 117 \\
\hline 30 & Av & Fr. dinar & $x x x$ & 0,84 & 10 & Prieto 117 \\
\hline 31 & Av & Fr. dinar & $x x x$ & 1,07 & 10 & Prieto 117 \\
\hline 32 & $A v$ & Fr. dinar & $x \times x$ & 0,52 & 9 & Prieto 117 \\
\hline 33 & $A v$ & Fr. dinar & $x x x$ & 0,84 & 11 & Prieto 117 \\
\hline 34 & $A v$ & Fr. dinar & $x x x$ & I,54 & 12 & Prieto 117 \\
\hline 35 & $A v$ & Fr. dinar & $x x x$ & 0,75 & 10 & Prieto 117 \\
\hline 36 & $A v$ & Fr. dinar & $x x x$ & 0,91 & 10 & Prieto 117 \\
\hline 37 & $A v$ & Fr. dinar & $x x x$ & 0,88 & 10 & Prieto 117 \\
\hline 38 & $A v$ & Fr. dinar & $x x x$ & 0,76 & 10 & Prieto 117 \\
\hline 39 & $A v$ & Fr. dinar & $x \times x$ & 0,93 & 10 & Prieto 117 \\
\hline 40 & $A v$ & Fr. dinar & $x x x$ & $0,7 \mid$ & II & Prieto 117 \\
\hline 41 & $A v$ & Fr, dinar & $x x x$ & 0,97 & 9 & Prieto 117 \\
\hline 42 & $A v$ & Fr. dinar & $x x x$ & 0,86 & 11 & Prieto 117 \\
\hline 43 & $A v$ & Fr. dinar & $x x x$ & 1,33 & 11 & Prieto 117 \\
\hline 44 & Av & Fr. dinar & $x \times x$ & 0,98 & 10 & Prieto 117 \\
\hline 45 & Av & Fr. dinar & $x \times x$ & 1,23 & 10 & Prieto 117 \\
\hline 46 & Av & Fr. dinar & $x \times x$ & 1,44 & 12 & Prieto 117 \\
\hline 47 & Av & Fr. dinar & $x x x$ & 0,46 & 8 & Prieto 117 \\
\hline 48 & Av & Fr. dinar & $x x x$ & 1,70 & 12 & Prieto 117 \\
\hline 49 & Av & Fr. dinar & $x \times x$ & 0,85 & 9 & Prieto 117 \\
\hline 50 & Av & Fr. dinar & $x x x$ & 0,90 & 10 & Prieto 117 \\
\hline 51 & Av & Fr. dinar & $x x x$ & 0,82 & 11 & Prieto 117 \\
\hline 53 & Av & Fr. dinar & $x x x$ & 0,93 & II & Prieto 117 \\
\hline 53 & $A v$ & Fr. dinar & $x x x$ & 0,64 & 12 & Prieto 117 \\
\hline 54 & Av & Fr. dinar & $x \times x$ & 0,80 & 11 & Prieto 117 \\
\hline 55 & $A v$ & Fr. dinar & $x \times x$ & 0,80 & 10 & Prieto 117 \\
\hline 56 & $A v$ & Fr. dinar & $x \times x$ & 1,10 & 10 & Prieto 117 \\
\hline
\end{tabular}


57 Av Fr. dinar $\quad \times 57 \quad$ I, I0 I । Prieto II6. La década se ve bien, pero de la unidad solo el final, que podría ser una ayn, por lo tanto un siete, nueve o cuatro.

\begin{tabular}{|c|c|c|c|c|c|c|}
\hline 58 & $A v$ & Fr. dinar & $x x x$ & $|, 2|$ & 13 & Prieto I I 6. \\
\hline 59 & Av & Fr. dinar & $x x x$ & 0,95 & 14 & Prieto 116. \\
\hline 60 & Av & Fr. dinar & $x x x$ & 0,63 & I | & Prieto I I 6. \\
\hline 61 & Av & Fr. dinar & $x x x$ & 1,10 & 10 & Prieto 116. \\
\hline 62 & Av & Fr. dinar & $x x x$ & 0,63 & 10 & Prieto I I6. \\
\hline 63 & Av & Fr. dinar & $x x x$ & 1,00 & 10 & Prieto I I 6. \\
\hline 64 & Av & Fr. dinar & $x x x$ & 0,57 & 10 & Prieto I I 6. \\
\hline 65 & Av & Fr. dinar & $x x x$ & 0,81 & 9 & Prieto I I 6. \\
\hline 66 & Av & Fr. dinar & $x x x$ & 0,74 & 9 & Prieto I I 6. \\
\hline 67 & Av & Fr. dinar & $x x x$ & 1,13 & 10 & Prieto 116 \\
\hline 68 & Av & Fr. dinar & $x x x$ & 0,86 & 10 & Prieto I 16. \\
\hline 69 & Av & Fr. dinar & $x x x$ & 0,86 & 10 & Prieto II 6. \\
\hline 70 & Av & Fr. dinar & $x x x$ & 1,22 & 10 & Prieto I I6. \\
\hline 71 & Av & Fr. dinar & $x x x$ & 1,15 & 10 & Prieto I I 6. \\
\hline 72 & Av & Fr. dinar & $x x x$ & 0,58 & 9 & Prieto 116. \\
\hline 73 & Av & Fr. dinar & $x x x$ & 0,95 & 10 & Prieto I I 6. \\
\hline 74 & Av & Fr. dinar & $x x x$ & 0,96 & II & Prieto 116 \\
\hline 75 & Av & Fr. dinar & $x x x$ & 0,56 & 9 & Prieto I 16. \\
\hline 76 & Av & Fr. dinar & $x x x$ & 1,10 & II & Prieto I I 6. \\
\hline 77 & Av & Fr. dinar & $x x x$ & 1,10 & 10 & Prieto I 16. \\
\hline 78 & Av & Fr. dinar & $46 x$ & 0,76 & 10 & Prieto I I 6. \\
\hline 79 & Av & Fr. dinar & $x x x$ & 0,63 & 10 & Prieto I I 6. \\
\hline 80 & Av & Fr. dinar & $x x x$ & 0,48 & 9 & Prieto I I6. \\
\hline 81 & Av & Fr. dinar & $x x x$ & 0,65 & 9 & Prieto 116 \\
\hline 82 & Av & Fr. dinar & $x x x$ & 0,82 & 10 & Prieto 116 \\
\hline 83 & Av & Fr. dinar & $x x x$ & 0,77 & 10 & Prieto 116. \\
\hline 84 & Av & Fr. dinar & $x x x$ & I,33 & 11 & Prieto 116. \\
\hline 85 & Av & Fr. dinar & $x x x$ & $|| 1$, & 11 & Prieto I I 6. \\
\hline 86 & Av & Fr. dinar & $x x x$ & 0,87 & 11 & Prieto I I 6. \\
\hline 87 & Av & Fr. dinar & $x x x$ & 1,22 & 10 & Prieto 116 \\
\hline 88 & Av & Fr. dinar & $x x x$ & 0,90 & 8 & Prieto $\mid 16$ \\
\hline 89 & Av & Fr. dinar & $x x x$ & 0,56 & 10 & Prieto || 6 \\
\hline 90 & $A v$ & Fr. dinar & $x x x$ & 0,96 & 11 & Prieto I I 6. \\
\hline 91 & Av & Fr. dinar & $x x x$ & 0,77 & 9 & Prieto I I 6. \\
\hline 92 & Av & Fr. dinar & $x x x$ & 1,23 & 11 & Prieto I I6. \\
\hline 93 & Av & Fr. dinar & $x x x$ & 0,77 & 11 & Prieto I I 6. \\
\hline 94 & Av & Fr. dinar & $x x x$ & 0,75 & 9 & Prieto 116 \\
\hline 95 & Av & Fr. dinar & $x x x$ & 0,84 & 10 & Prieto | | \\
\hline 96 & Av & Fr. dinar & $x x x$ & 1,02 & 10 & Prieto I I \\
\hline 97 & Av & Fr. dinar & $x x x$ & 0,79 & 10 & Prieto I I \\
\hline 98 & Av & Fr. dinar & $x x x$ & 1,01 & 11 & Prieto I 16. \\
\hline 99 & $A v$ & Fr. dinar & $x x x$ & 0,64 & 10 & Prieto 116 \\
\hline 100 & $A v$ & Fr. dinar & $x x x$ & 0,63 & 10 & Prieto 116 \\
\hline 101 & Av & Fr. dinar & $x x x$ & $0,7 \mid$ & 10 & Prieto 116 \\
\hline 102 & Av & Fr. dinar & $x x x$ & 0,93 & 10 & Prieto ||$\epsilon$ \\
\hline 103 & Av & Fr. dinar & $x x x$ & 1,02 & 10 & Prieto I I \\
\hline 104 & Av & Fr. dinar & $x x x$ & 0,87 & 7 & Prieto | | \\
\hline 105 & Av & Fr. dinar & 457 & 1,06 & 11 & Prieto 116. \\
\hline
\end{tabular}




\begin{tabular}{|c|c|c|c|c|c|}
\hline 106 & Fr. dinar & $x x x$ & 1,48 & 10 & Prieto 116. \\
\hline 107 & Fr. dinar & $x x x$ & I,04 & II & Prieto I I6. \\
\hline 108 & Fr. dinar & $x x x$ & 0,83 & 9 & Prieto I I6. \\
\hline 109 & Fr. dinar & $x x x$ & 0,84 & 9 & Prieto 116. \\
\hline 110 & Fr. dinar & $x x x$ & 0,62 & 10 & Prieto 116. \\
\hline III & Fr. dinar & $x x x$ & 1,23 & II & Prieto I I6. \\
\hline 112 & Fr. dinar & $x x x$ & 0,88 & 10 & Prieto I I 6. \\
\hline 113 & Fr. dinar & $x x x$ & 0,91 & 10 & Prieto I I6. \\
\hline 114 & Fr. dinar & $x x x$ & 1,06 & 10 & Prieto I I6. \\
\hline 115 & Fr. dinar & $x x x$ & 1,17 & 11 & Prieto 116. \\
\hline 116 & Fr. dinar & $x x x$ & 1,26 & II & Prieto I I6. \\
\hline 117 & Fr. dinar & $x x x$ & 1,05 & 10 & Prieto I I 6. \\
\hline 118 & Fr. dinar & $x x x$ & 1,10 & 9 & Prieto I I6. \\
\hline 119 & Fr. dinar & $x x x$ & ।,38 & 10 & Prieto I I6. \\
\hline 120 & Fr. dinar & $x x x$ & 0,94 & 10 & Prieto I I6. \\
\hline 121 & Fr. dinar & $x x x$ & 0,76 & 10 & Prieto 116. \\
\hline 122 & Fr. dinar & $x x x$ & I,04 & II & Prieto I I 6. \\
\hline 123 & Fr. dinar & $x x x$ & 1,18 & II & Prieto 116. \\
\hline 124 & Fr. dinar & $x x x$ & 0,95 & 10 & Prieto I I 6. \\
\hline 125 & Fr. dinar & $x x x$ & 1,52 & 13 & Prieto I I6. \\
\hline 126 & Fr. dinar & $x x x$ & 0,80 & 9 & Prieto I I6. \\
\hline 127 & Fr. dinar & $x x x$ & $0,7 \mid$ & 9 & Prieto I I6. \\
\hline 128 & Fr. dinar & $x x x$ & 1,02 & 9 & Prieto I I6. \\
\hline 129 & Fr. dinar & $x x x$ & 0,82 & II & Prieto I I6. \\
\hline 130 & Fr. dinar & $x x x$ & 0,80 & 9 & Prieto I I6. \\
\hline 131 & Fr. dinar & $x x x$ & 1,32 & 13 & Prieto I I6. \\
\hline 132 & Fr. dinar & $45 x$ & 0,80 & 10 & Prieto I 16. \\
\hline 133 & Fr. dinar & $x x x$ & $|, 0|$ & 10 & Prieto I I6. \\
\hline 134 & Fr. dinar & $x x x$ & 0,53 & 10 & Prieto 116. \\
\hline 135 & Fr. dinar & $x x x$ & 0,88 & 10 & Prieto I I6. \\
\hline 136 & Fr. dinar & $x x x$ & 1,28 & 13 & Prieto I I6. \\
\hline 137 & Fr. dinar & $x x x$ & $0,7 \mathrm{I}$ & II & Prieto 116. \\
\hline 138 & Fr. dinar & $x x x$ & 1,00 & 10 & Prieto I I6. \\
\hline 139 & Fr. dinar & $x x x$ & 0,66 & 10 & Prieto I I6. \\
\hline 140 & Fr. dinar & $x x x$ & 0,88 & 10 & Prieto I I 6. \\
\hline $14 \mid$ & Fr. dinar & $x x x$ & 0,55 & 8 & Prieto I I6. \\
\hline 142 & Fr. dinar & $x x x$ & 0,91 & 12 & Prieto I I6. \\
\hline 143 & Fr. dinar & $x x x$ & 0,63 & II & Prieto I I6. \\
\hline 144 & Fr. dinar & $x x x$ & $|| \mid$, & 10 & Prieto I I 6. \\
\hline 145 & Fr. dinar & $x x x$ & 0,60 & 10 & Prieto 116. \\
\hline 146 & Fr. dinar & $x x x$ & 0,73 & 10 & Prieto 116. \\
\hline 147 & Fr. dinar & $x x x$ & 0,70 & 9 & Prieto 116. \\
\hline 148 & Fr. dinar & $x x x$ & 0,74 & 10 & Prieto 116. \\
\hline 149 & Fr. dinar & $x x x$ & 0,57 & 10 & Prieto I I6. \\
\hline 150 & Fr. dinar & $x x x$ & 0,79 & 8 & Prieto 116. \\
\hline$|5|$ & Fr. dinar & $x x x$ & 0,84 & II & Prieto I I 6. \\
\hline 152 & Fr. dinar & $x x x$ & $|, 0|$ & 14 & Prieto I I6. \\
\hline 153 & Fr. dinar & $x x x$ & 1,16 & II & Prieto I I 6. \\
\hline 154 & Fr. dinar & $x x x$ & 0,55 & 9 & Prieto I I6. \\
\hline 155 & Fr. dinar & $x x x$ & 0,86 & 10 & Prieto I 16. \\
\hline 156 & Fr. dinar & $x x x$ & 2,30 & 14 & Prieto 116. \\
\hline
\end{tabular}




\begin{tabular}{|c|c|c|c|c|c|c|}
\hline 157 & $A v$ & Fr. dinar & $x x x$ & 1,18 & 11 & Prieto 116 \\
\hline 158 & Av & Fr. dinar & $x \times 1$ & 0,86 & 10 & Prieto 116 \\
\hline 159 & $A v$ & Fr. dinar & $x X x$ & 0,80 & 9 & Prieto 116 \\
\hline 160 & $A v$ & Fr. dinar & $x x x$ & 0,44 & 8 & Prieto 116 \\
\hline 161 & $A v$ & Fr. dinar & $x x x$ & 1,15 & 10 & Prieto 116 \\
\hline 162 & Av & Fr. dinar & $x x x$ & 1,36 & 13 & Prieto 116 \\
\hline 163 & $A v$ & Fr. dinar & $x x x$ & 1,20 & 12 & Prieto 116 \\
\hline 164 & $A v$ & Fr. dinar & $x x x$ & 0,93 & 10 & Prieto 116 \\
\hline 165 & $A v$ & Fr. dinar & $x x x$ & 1,04 & 13 & Prieto 116 \\
\hline 166 & $A v$ & Fr. dinar & $x x x$ & ।,38 & 12 & Prieto 116 \\
\hline 167 & $A v$ & Fr. dinar & $x \times x$ & 1,20 & $\mid 1$ & Prieto 116 \\
\hline & \multicolumn{6}{|c|}{ Linea de puntos entre las dos lineas circulares de separación del reverso } \\
\hline 168 & Av & Fr. dinar & $x x x$ & 0,91 & 10 & Prieto 116 \\
\hline 169 & Av & Fr. dinar & $x x x$ & 0,70 & 9 & Prieto 116 \\
\hline |7| & Av & Fr. dinar & $x x x$ & 0,77 & 10 & Prieto 116 \\
\hline |7| & Av & Fr. dinar & $x \times x$ & 1,02 & II & Prieto $\mid 16$ \\
\hline 172 & Av & Fr. dinar & $x x x$ & 1,00 & 10 & Prieto 116 \\
\hline 173 & $A v$ & Fr. dinar & $x x x$ & 0,83 & 9 & Prieto 116 \\
\hline 175 & Av & Fr. dinar & $x x x$ & 0,83 & II & Prieto 116 \\
\hline 175 & Av & Fr. dinar & $x \times 7$ & 1,38 & 10 & Prieto 116 \\
\hline 176 & $A v$ & Fr. dinar & $x x x$ & 1,14 & 10 & Prieto 116 \\
\hline 177 & $A v$ & Fr. dinar & $x x x$ & 0,72 & II & Prieto 116 \\
\hline 178 & $A v$ & Fr. dinar & $x x x$ & 0,62 & 10 & Prieto 116 \\
\hline 179 & $A v$ & Fr. dinar & $x x x$ & 0,70 & 9 & Prieto 116 \\
\hline
\end{tabular}

Banū Yahwar de Córdoba

Abū'I-Walīd Muḥammad b. Yahwar (434-456 H.) (Qurțuba )

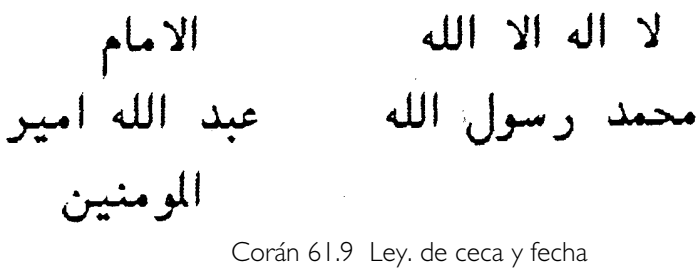

180 Av Fr. dinar $\quad x \times x \quad 0,70$ Fecha ilegible y ceca asimismo. Punto sobre leyenda de anverso

\begin{tabular}{|c|c|c|c|c|c|}
\hline |8| & Fr. dinar & $x x x$ & 0,57 & 10 & Prieto 352a var. \\
\hline 182 & Fr. dinar & $x x x$ & 0,74 & 9 & Prieto 352a, var. \\
\hline 183 & Fr. dinar & $x x x$ & 1,10 & 10 & Prieto 352a, var. \\
\hline 184 & Fr. dinar & $x x x$ & 0,76 & 9 & Prieto 352a, var. \\
\hline 185 & Fr. dinar & $x x x$ & $0,7 \mid$ & 9 & Prieto 352a, var. \\
\hline 186 & Fr. dinar & $x x x$ & 0,88 & 10 & Prieto 352a, var. \\
\hline Av & Fr. dinar & $x x x$ & 0,58 & 9 & Prieto 352a, var. \\
\hline 188 & Fr. dinar & $x x x$ & 0,59 & 9 & Prieto 352a, var. \\
\hline $\begin{array}{l}\text { Av } \\
\text { Se o }\end{array}$ & \multicolumn{5}{|c|}{ Se observa parte de la decena del numeral cincuenta. } \\
\hline$A v$ & Fr. dinar & $x x x$ & 0,92 & 9 & Prieto $352 \mathrm{a}$, var. \\
\hline$A v$ & Fr. dinar & $x x x$ & 0,56 & 9 & Prieto 352a, var. \\
\hline Av & Fr. dinar & $x x x$ & 0,73 & 9 & Prieto 352a, var. \\
\hline
\end{tabular}




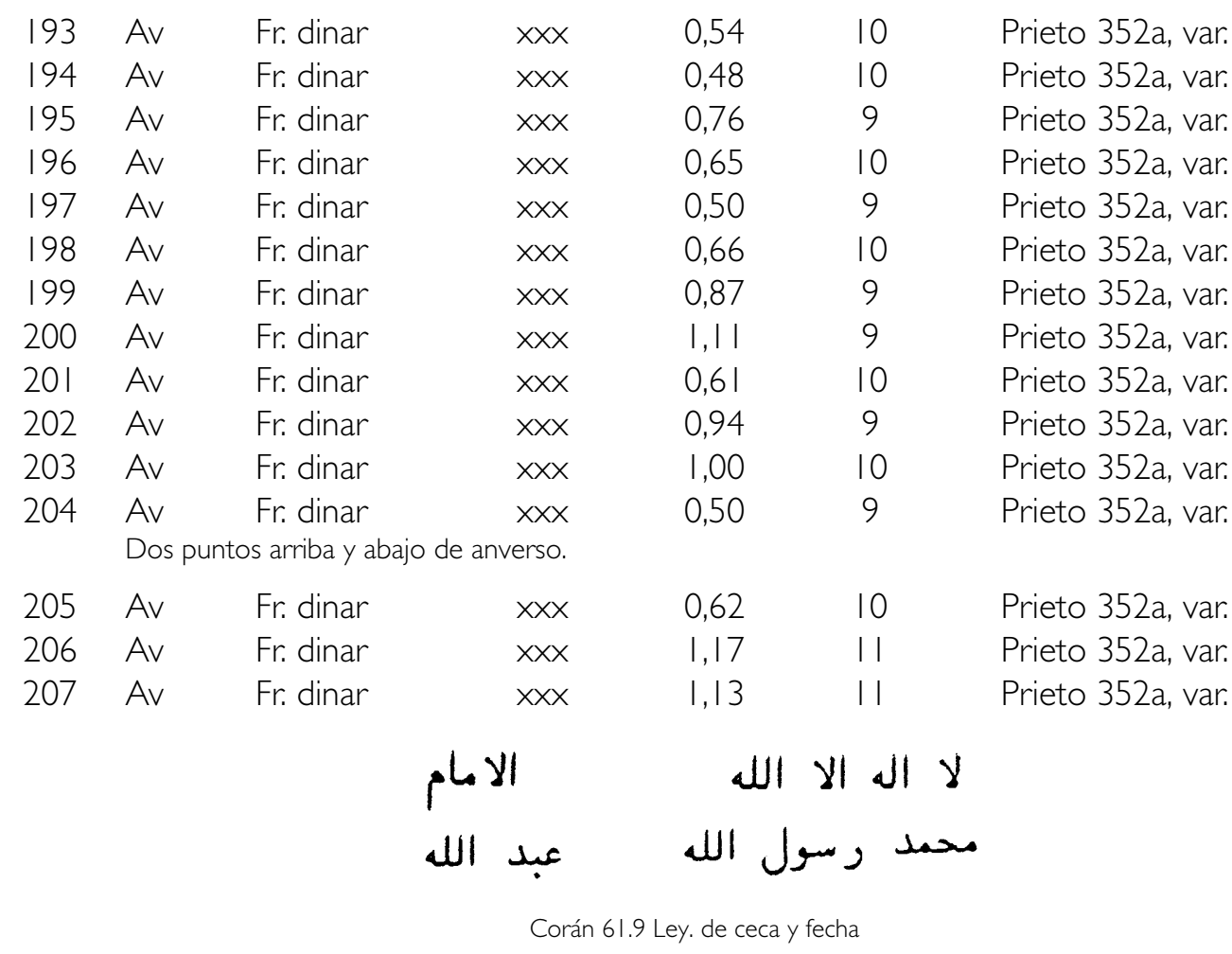

208 Av Fr. dinar $\quad x \times x \quad 0,53 \quad 9 \quad$ Inédita.

El anverso corresponde a la Prieto 352, y el reverso a Prieto 353. Como decoracin presenta puntos encima y debajo del anverso y, en el reverso, solo debajo.

Por las razones ya comentadas creo que podría atribuirse a la serie de Córdoba de los Banu Yahwar.

209 Av Fr. dinar $\quad x \times x \quad$ I,I0 II Inédita.

Decoración de dos palmetas en anverso y reverso, zona superior presente en toda la serie, junto con ápices florales de ceca en anverso; pueden leerse algunos trazos de al-Andalus, pero muy dudosos.

\begin{tabular}{|c|c|c|c|c|c|}
\hline $210 \quad A v$ & Fr. dinar & $x \times 3$ & 0,66 & 9 & Inédita \\
\hline 211 & Fr. dinar & $x x x$ & 0,72 & 10 & Inédita \\
\hline 212 & Fr. dinar & $x x x$ & 0,68 & 9 & Inédita \\
\hline 213 & Fr. dinar & $x x x$ & 0,64 & 10 & Inédita \\
\hline 214 & Fr. dinar & $x x x$ & 0,70 & 10 & Inédita \\
\hline 215 & Fr. dinar & $x x x$ & 0,52 & 9 & Inédita \\
\hline 216 & Fr. dinar & $x x x$ & 0,41 & 9 & Inédita \\
\hline 217 & Fr. dinar & $x x x$ & 0,73 & 10 & Inédita \\
\hline 218 & Fr. dinar & $x x x$ & 0,62 & | & Inédita \\
\hline 219 & Fr. dinar & $x x x$ & 1,14 & 10 & Inédita \\
\hline 220 & Fr. dinar & $x x x$ & 0,53 & 9 & Inédita \\
\hline 221 & Fr. dinar & $x \times x$ & 0,32 & 9 & Inédita \\
\hline & \multicolumn{5}{|c|}{ Punto arriba y dos debajo en el reverso. } \\
\hline 222 & Fr. dinar & $x x x$ & 0,76 & 10 & Inédita \\
\hline$A v$ & Fr. dinar & $x x x$ & 1,13 & 11 & Inédita \\
\hline$A v$ & Fr. dinar & $x x x$ & 0,71 & 9 & Inédita \\
\hline 225 & Fr. dinar & $x x x$ & 0,78 & 9 & Inédita \\
\hline 226 & Fr. dinar & $x x x$ & 0,40 & 9 & Inédita \\
\hline 227 & Fr. dinar & $x x x$ & 0,53 & 9 & Inédita \\
\hline Av & Fr. dinar & $x x x$ & 0,72 & 9 & Inédita \\
\hline
\end{tabular}




\begin{tabular}{|c|c|c|c|c|c|c|}
\hline 229 & $A v$ & Fr. dinar & $x x x$ & 0,97 & 11 & Inédita. \\
\hline 230 & $A v$ & Fr. dinar & $x x x$ & 0,52 & 10 & Inédita. \\
\hline 231 & $A v$ & Fr. dinar & $x x x$ & 0,73 & 10 & Inédita. \\
\hline 232 & $A v$ & Fr. dinar & $x x x$ & 0,60 & 9 & Inédita. \\
\hline 233 & $A v$ & Fr. dinar & $X X X$ & I,08 & 10 & Inédita. \\
\hline 234 & $A v$ & Fr. dinar & $x x x$ & 0,95 & 10 & Inédita. \\
\hline 235 & $A v$ & Fr. dinar & $x x x$ & 0,70 & I I & Inédita. \\
\hline 236 & $A v$ & Fr. dinar & $x x x$ & 0,51 & 10 & Inédita. \\
\hline 237 & $A v$ & Fr. dinar & $x x x$ & 0,69 & 10 & Inédita. \\
\hline 238 & $A v$ & Fr. dinar & $x x x$ & 0,61 & 9 & Inédita. \\
\hline 239 & $A v$ & Fr. dinar & $x x x$ & 0,56 & 9 & Inédita. \\
\hline 240 & Av & Fr. dinar & $x x x$ & 0,64 & 9 & Inédita. \\
\hline 241 & $A v$ & Fr. dinar & $x x x$ & 0,72 & 10 & Inédita. \\
\hline 242 & $A v$ & Fr. dinar & $x x x$ & 0,72 & 10 & Inédita. \\
\hline 243 & $A v$ & Fr. dinar & $x x x$ & 1,14 & I | & Inédita. \\
\hline 244 & $A v$ & Fr. dinar & $x x x$ & 0,54 & 9 & Inédita. \\
\hline 245 & $A v$ & Fr. dinar & $x x x$ & 0,77 & 11 & Inédita. \\
\hline 246 & $A v$ & Fr. dinar & $x x x$ & $0,7 \mid$ & 9 & Inédita. \\
\hline 247 & $A v$ & Fr. dinar & $x x x$ & 0,85 & 11 & Inédita. \\
\hline 248 & $A v$ & Fr. dinar & $x X x$ & 0,75 & 10 & Inédita. \\
\hline 249 & $A v$ & Fr. dinar & $x x x$ & 0,79 & 10 & Inédita. \\
\hline 250 & $A v$ & Fr. dinar & $x x x$ & 0,67 & 10 & Inédita. \\
\hline 251 & $A v$ & Fr. dinar & $x x x$ & 0,60 & 8 & Inédita. \\
\hline 252 & $A v$ & Fr. dinar & $x x x$ & 0,91 & 9 & Inédita. \\
\hline 253 & $A v$ & Fr. dinar & $x x x$ & I,08 & I I & Inédita. \\
\hline 254 & $A v$ & Fr. dinar & $x \times 9$ & $|, 2|$ & 12 & Inédita. \\
\hline 255 & $A v$ & Fr. dinar & $x x x$ & 0,94 & 10 & Inédita. \\
\hline 256 & $A v$ & Fr. dinar & $x x x$ & 1,36 & 11 & Inédita. \\
\hline 257 & $A v$ & Fr. dinar & $x x x$ & 0,65 & 12 & Inédita. \\
\hline 258 & $A v$ & Fr. dinar & $x x x$ & 0,90 & 10 & Inédita. \\
\hline 259 & $A v$ & Fr. dinar & $x \times 3$ & 0,94 & 10 & Inédita. \\
\hline 260 & $A v$ & Fr. dinar & $x x x$ & 1,05 & 9 & Inédita. \\
\hline 261 & $A v$ & Fr. dinar & $x x x$ & 0,98 & I I & Inédita. \\
\hline 262 & $A v$ & Fr. dinar & $x x x$ & 0,58 & 9 & Inédita. \\
\hline 263 & $A v$ & Fr. dinar & $x x x$ & 0,79 & 10 & Inédita. \\
\hline 264 & $A v$ & Fr. dinar & $x x x$ & $|, 4|$ & 12 & Inédita. \\
\hline 265 & $A v$ & Fr. dinar & $x x x$ & 0,38 & 9 & Inédita. \\
\hline 266 & $A v$ & Fr. dinar & $x x x$ & I,07 & 12 & Inédita. \\
\hline 267 & $A v$ & Fr. dinar & $x x x$ & 0,91 & 11 & Inédita. \\
\hline 268 & $A v$ & Fr. dinar & $x x x$ & 0,74 & 9 & Inédita. \\
\hline 269 & $A v$ & Fr. dinar & $x x x$ & 0,73 & 9 & Inédita. \\
\hline 270 & $A v$ & Fr. dinar & $x x x$ & 0,72 & 9 & Inédita. \\
\hline 27I & $A v$ & Fr. dinar & $x x x$ & 0,74 & 9 & Inédita. \\
\hline 272 & $A v$ & Fr. dinar & $x x x$ & 0,97 & II & Inédita. \\
\hline 273 & $A v$ & Fr. dinar & $x x x$ & 0,62 & 9 & Inédita. \\
\hline 274 & $A v$ & Fr. dinar & $x x x$ & 0,81 & 10 & Inédita. \\
\hline
\end{tabular}




\section{BIBLIOGRAFÍA}

BARCELO, Miquel (1988): "Coins from afar? New evidence on coin production and fiscal administrative practice in the Late Umayyad Caliphate", Problems of Medieval Coinage in Iberian Peninsule 3, Santarem, pp. I07-I I 8.

BARCELO, Miquel (1985): "Un estudio sobre la estructura fiscal y procedimientos contables del Emirato Omeya de Córdoba (|38-300/755-9|2) y el Califato (300-366/9 I2-976)", Acta Medievalia, 5-6.

BARCELO, Miquel ( 1992 ): "Rodes que girens dins el foc de le infern" o per a qué servia la moneda dels taifes", Gaceta Numismática, $105-106$, 15-24.

CANTO GARCIA, Alberto (1992): "Hallazgos monetarios en el periodo taifa", Gaceta Numismática, I05-106, 25-42.

\section{RESUMEN}

El artículo presenta de manera sucinta las diferencias entre el modelo de circulación de época omeya en al-Andalus frente al que se plantea en el periodo taifa. Como ejemplo se presentan dos hallazgos provenientes de Jaén.

PALABRAS CLAVE: Al-Andalus, Numismática, Hallazgos Jaén
CANTO GARCIA, Alberto (1994): "La moneda", Los Reinos de Taifas. Al-Andalus en el siglo XI. Historia de España, dir. R. Menéndez Pidal., Madrid, pp. 275-297

CHALMETA, Pedro (1987-88): "Monnaie de recouvrement des impots et taux de change dans al-Andalus", Quaderni Studi Arabi, 5-6, pp. 153-166.

PRIETO VIVES, Antonio (1926): Los Reyes de Taifas. Estudio histórico-numismático de los musulmanes españoles en el siglo V de la Hégira (XI d.C.), Madrid.

SAENZ-DIEZ, J.I.: "Dos hallazgos Hispanoárabes en Museos Nacionales: "Cruz Conde" (Arqueológico Nacional) y "Haza del Carmen" (Arqueológico de Córdoba)", Gaceta Numismática 74-75, pp. |47-I52.

\section{ABSTRACT}

The paper show in a short manner the main differences beetwen the pattern of monetary circulation in the Umayad period in Al-Andalus in contrast with the Party Kingdoms. Like a sample two hoards of Xlth, provening from Jaén, are published. 


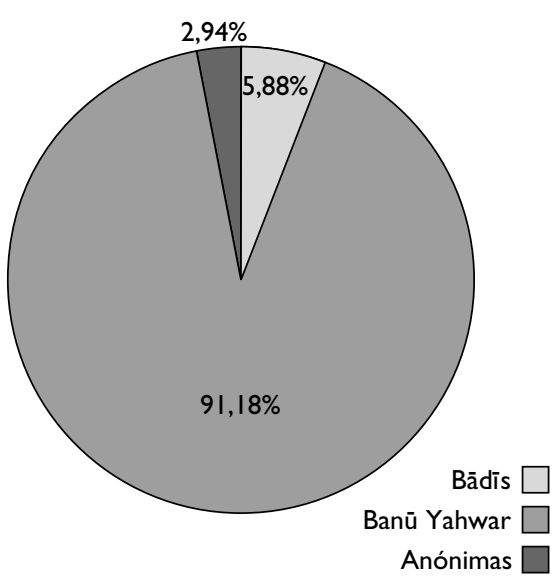

Fig. I

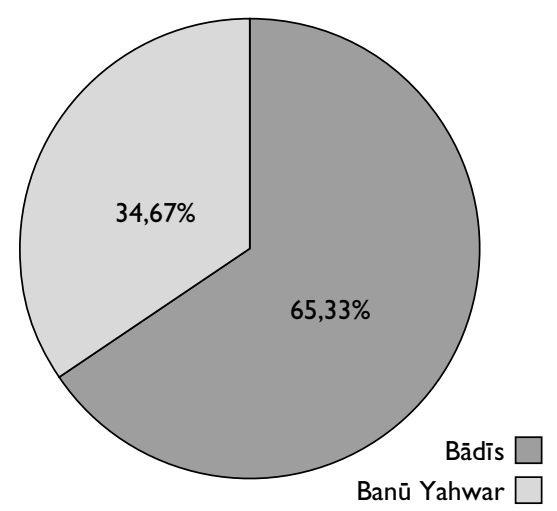

Fig. 2

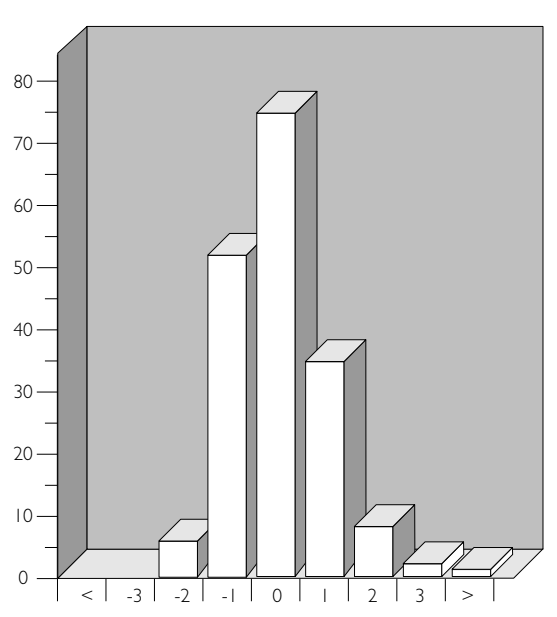

Fig. 3. Histograma de intervalos de desviación típica. Serie zirí

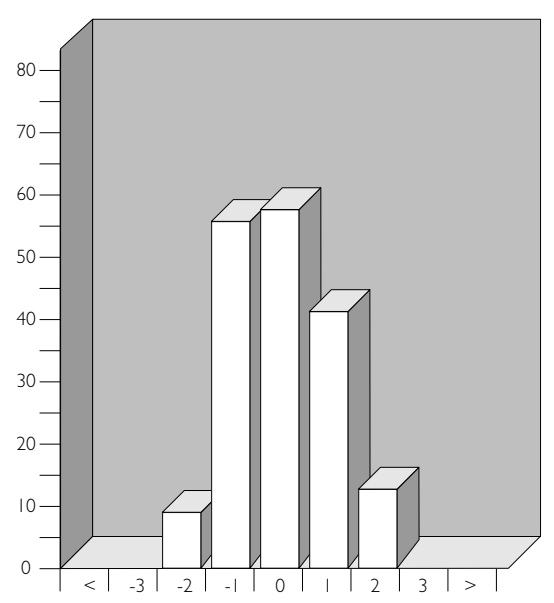

Fig. 5. Histograma de intervalos de desviación típica. Serie de Banū Yahwar

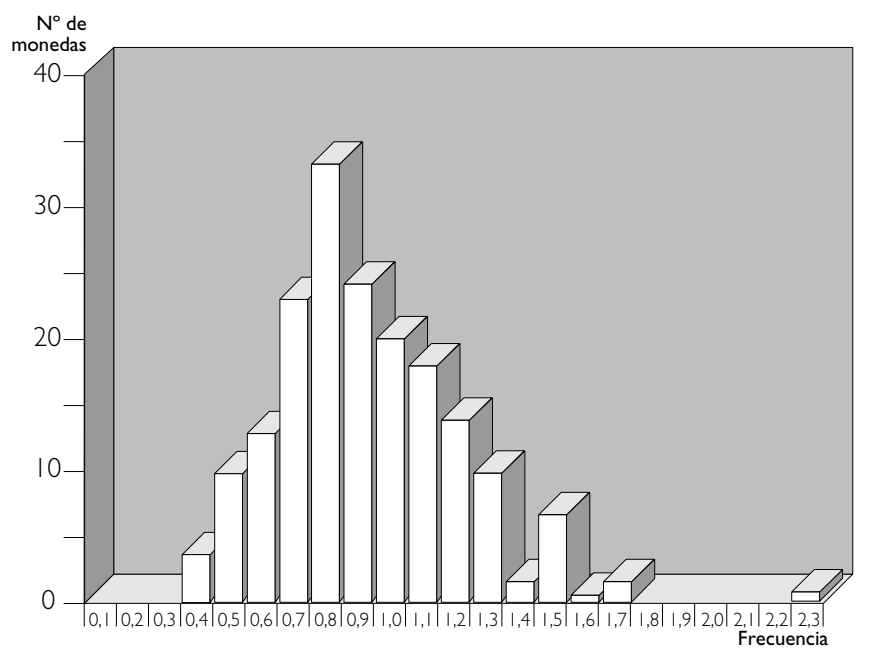

Fig. 4. Frecuencia de pesos de la serie zirí

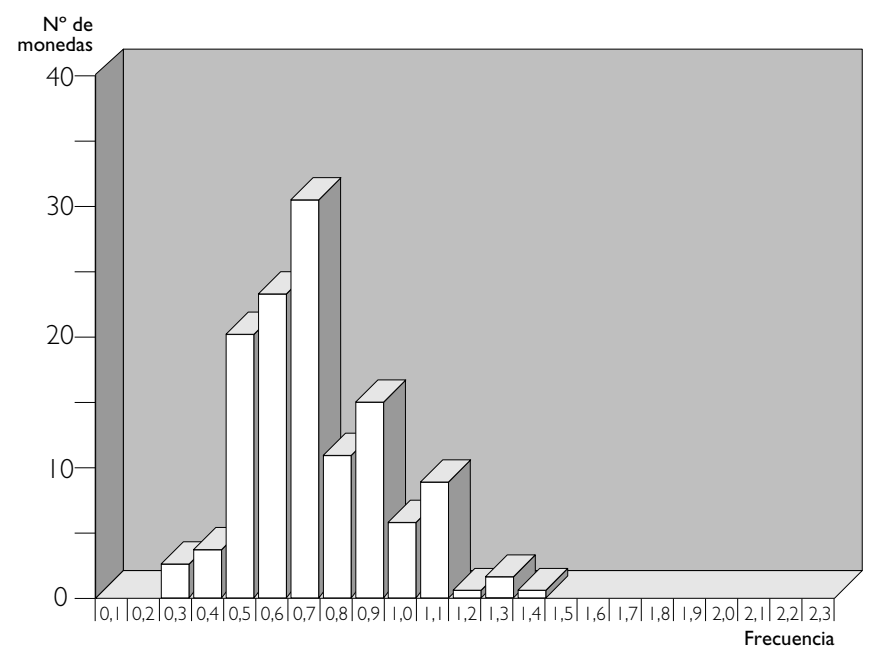

Fig. 6. Frecuencia de pesos de la serie B. Yahwar 